\title{
PROPERTIES OF THE SYMBOL OF MULTIDIMENSIONAL SINGULAR INTEGRALS IN THE WEIGHTED SPACES AND OSCILLATING MULTIPLIERS
}

\begin{abstract}
AKIF D. GADJIEV
Abstract. Differential properties of symbols of multidimensional singular integrals in the weighted space of Bessel potentials on the sphere with the weighted functions, having singularities on a sphere are studied. The main results are applied to obtaining theorems on Fourier multipliers of spherical harmonic expansions.
\end{abstract}

Mathematics subject classification (2010): 42B15, 42B25, 42B35.

Keywords and phrases: Symbols, Beltrami operator, spherical harmonics, Hardy inequalities, multipliers, the space of potentials.

\section{REFERENCES}

[1] S. G. Mikhlin, Multidimensional singular integrals and integral equations, Fizmatgiz, Moscow, 1962, English transl., Pergamon Press, 1965.

[2] Elias M. Stein, Singular integral operators and differentiability properties of functions, Princeton Univ. Press, Princeton, State N.J., 1970.

[3] S. G. Mikhlin, S. Pressdorf, Singular integral operators, Springer Verlag, Berlin Heidelberg, New York-Tokyo, 1986.

[4] S. G. Samko, Singular integral on the sphere and the construction characteristics by symbol, Izv. VUZ ov Mathematics, No. 4 (251) (1983), 28-42. (Russian)

[5] A. D. GADJIEV, Exact theorems on multipliers of spherical expansions and some applications, in "Special problems of function theory", issue 4, Baku, Elm, 1989, 73-100. (Russian)

[6] A. D. GADJIEv, Multipliers of Fourier Series in spherical functions and properties of the symbol of the multidimensional singular operators, Dokl. Akad. Nauk USSR, 266, 2 (1982); English transl. Soviet Math. Dokl. 26, 2 (1982).

[7] A. D. GADZIEV, On a connection between the properties of the symbol and the characteristic of a multidimensional singular integral operator in $L_{p}$-spaces, Dokl. Akad. Nauk USSR 250 (1980), 531-534. English transl. in Soviet Math. Dokl. 21 (1980).

[8] A. D. GADZIEV, On differentiability properties of the symbol of a multidimensional singular integral operator, Matem. Sbornik 114(156), 4 (198); English transl. Math. USSR Sbornik 42, 4 (1982), $427-$ 450.

[9] F. G. Maksudov, A. D. GadjIev, Spherical convolutions and oscillating multipliers, Baku, Elm, 2000, 1-159. (Russian)

[10] YU. N. SKIBA, Instability of the Rossby - Haurwitz wave in the invariant sets of perturbations, J. Math. Anal. Appl. 290 (2004), 686-701.

[11] YU. N. SKIBA, On the spectral problem in the linear stability study of flows on a sphere, J. Math. Anal. Appl. 270 (2002), 165-180.

[12] Boris RUBIN, Inversion and characterization of the hemispherical transform, J. D'Anal. Math. 77 (1999), 105-128.

[13] Boris Rubin, Notes on Radon Transforms in integral geometry, Fract. Calc. Appl. Anal. 6, 1 (2003), $25-72$.

[14] KH. P. Rustamov, On the smoothness of the symbol of multidimensional singular integral operators, Analysis Math. 13 (1987), 93-199. 
[15] V. S. KRYUCHKOV, Study of differential properties of symbol of Kalderon - Zygmund singular integral, $\mathrm{PhD}$ Dissertation, Moscow, 1983 (Russian).

[16] B. G. VAKULOV, Spherical operators of potential type in generalized Holder spaces on the sphere, PhD Dissertation, Rostov on Don, 1986 (Russian).

[17] P. M. PAVlov, Spherical hypersingular integrals and its applications, PhD Dissertation, Rostov on Don, 1987 (Russian).

[18] Robert S. STRICHARTZ, Convolution with kernels having singularities on a sphere, Trans. American Math. Soc. 148 (1970), 461-471.

[19] V. S. KRYUChKov, Differential properties of the symbol of a Calderon-Zygmund singular integral operator, Proc. of the Steklov Inst. of Math. 170, 1 (1987), 169-183 (Russian). 